\title{
硅基芯片表面化学性质对蛋白质固定化的影响
}

\author{
周稳稳 1,2 廉 洁 1,2 胡科家 1,2 高云华 $1, *$ 徐 百 1 , * \\ ( ${ }^{1}$ 中国科学院理化技术研究所光化学转换和功能材料重点实验室, 北京 $100190 ;{ }^{2}$ 中国科学院研究生院, 北京 100049)
}

\begin{abstract}
摘要： 制备蛋白质芯片的关键在于将蛋白质固定到芯片表面并保持其生物学活性. 本实验中, 我们分别采用物 理吸附、直接化学固定、加人间隔臂化学固定和生物亲和作用固定的方法将癌肧抗原(CEA)抗体固定到硅基芯片 的二氧化硅表面. 基于抗原-抗体的特异性相互作用, 利用双抗体夹心酶联免疫法(ELISA)评价各种方法固定抗 体的效果. 实验结果表明, 在修饰有氨基的表面采用戊二醛作为偶联试剂固定 CEA 抗体具有最高的偶联效率, 引人多聚赖氨酸(poly-L-lysine)作为间隔臂可以显著增强固定效果, 并可进一步降低非特异性吸附. 而利用生物 亲和作用固定 CEA 抗体也可获得较好的固定效果, 但是非特异性吸附较严重.
\end{abstract}

关键词：蛋白质芯片；抗体固定；夹心反应；酶联免疫吸附分析；氨基表面；戊二醛；多聚赖氨酸 中图分类号: 0647

\section{Effect of Surface Chemical Properties of a Silicon Chip on Antibody Immobilization}

\author{
ZHOU Wen-Wen ${ }^{1,2} \quad$ LIAN Jie $^{1,2} \quad$ HU Ke-Jia $^{1,2} \quad$ GAO Yun-Hua ${ }^{1, *} \quad$ XU Bai ${ }^{1, *}$ \\ ( ${ }^{1}$ Key Laboratory of Photochemical Conversion and Optoelectronic Materials, Technical Institute of Physics and Chemistry, \\ Chinese Academy of Sciences, Beijing 100190, P. R. China; ${ }^{2}$ Graduate University of Chinese Academy of Sciences, \\ Beijing $\quad 100049$, P. R. China)
}

\begin{abstract}
The key to constructing a protein microarray is the stable immobilization of proteins and the retention of their biological activities. In this study, immobilization of the carcinoembryonic antigen (CEA) antibody onto a silicon dioxide surface was investigated by physical adsorption, direct chemical covalent conjugation, spacer-added chemical covalent conjugation, and biological affinity interactions. Based on the specific antibody-antigen interactions, the sandwich reaction, enzyme-linked immunosorbent assay (ELISA) was chosen to evaluate various immobilization strategies. The most efficient immobilization strategy was with glutaraldehyde as a coupling reagent between the CEA antibody and the amino surface. The attachment of a spacer-arm comprising poly-L-lysine significantly improved the immobilization efficiency and simultaneously decreased nonspecific adsorption. High immobilization efficiency and stronger nonspecific adsorption were also observed when the CEA antibody was immobilized by bioaffinity interactions.
\end{abstract}

Key Words : Protein microarray; Antibody immobilization; Sandwich reaction; Enzyme-linked immunosorbent assay; Amino surface; Glutaraldehyde; Poly-L-lysine

蛋白质芯片可用于研究蛋白质分子之间的相互 作用, 在分子识别、临床诊断、环境监测、质量控制、 高通量和快速检测领域有着广泛的应用前景 $[1-6]$. 构
建蛋白质芯片的基础是将特异性的蛋白质分子(如 抗体分子)固定到芯片表面并保持其生物学活性. 由 于蛋白质在结构上和化学性质上都比 DNA 更复

Received: April 22, 2010; Revised: July 9, 2010; Published on Web: September 3, 2010.

"Corresponding authors. GAO Yun-Hua, Email: yhgao@mail.ipc.ac.cn; Tel: +86-10-82543581. XU Bai, Email: nanobioic@ gmail.com.

The project was supported by the National Natural Science Foundation of China (20975106), Funds of the Chinese Academy of Sciences for Key Topics in Innovation Engineering (KJCX2-YW-M15) and Important National Science \& Technology Specific Projects, China (2008ZX08012-001). 国家自然科学基金(20975106), 中国科学院知识创新工程重要方向项目(KJCX2-YW-M15)及国家重大专项(2008ZX08012-001)资助 
杂 ${ }^{[7-8]}$, 在固定到固体表面的过程中容易失活,一般 认为这可能是由于固相表面的化学微环境因素导致 了蛋白质空间结构的改变 ${ }^{[9-11]}$. 此外, 化学的、物理学 的以及结构上的种种差异使得各种蛋白质在固体表 面上的固定效率呈现很大差异 ${ }^{[12-13]}$. 因此对芯片表 面蛋白质固定策略的研究是极其重要的. 如何在不 同的固相表面固定足够多的保持生物活性的蛋白质 分子是具有挑战性的工作, 也是众多蛋白质组学研 究者孜孜追求的目标 ${ }^{[14-20]}$, 许多研究者开发出各种 新颖的蛋白质固定技术, 并取得了较好的效果.

表面化学在蛋白质固定中扮演了一个极其重要 的角色, 各种固定蛋白质的策略都离不开对固相表 面的化学修饰. 首先需要在固相表面(如硅片、玻片、 塑料等)衍生出化学活性的功能团, 然后通过化学反 应将蛋白质分子(如抗体分子)偶联固定到这种化学 活化的固相表面. 在这个过程中, 如何提高偶联的效 率并保持蛋白质分子的生物活性是主要挑战. 目前 已经有许多不同的表面处理方法和共价偶联方 法 ${ }^{[12,14-15,21]}$. 这些方法大致可以分为四类: (a) 直接物 理吸附 ${ }^{[22-24]}$, 如在聚苯乙烯表面和聚赖氨酸表面直 接吸附蛋白质分子; (b) 直接共价连接 ${ }^{[15,25]}$, 如在短链 氨基、颈基或羧基表面直接偶联蛋白质分子; (c) 加 人间隔臂共价连接 ${ }^{[26-29]}$, 即在偶联蛋白质分子之前, 先引人一层高分子, 通常选择树枝状高分子或具有 较好生物相容性的高聚物; (d) 利用生物亲和作用固 定蛋白质分子 [17,30-31], 如先将结合蛋白 A 固定到固相 表面, 然后再利用它们与免疫球蛋白 Fc 段的特异性 结合固定抗体分子. 虽然已经有很多文献报道了上 述不同的表面修饰方法, 但是很少有文献系统研究 这些方法对蛋白质固定化效果的影响. 因此, 我们设 计了一个系统研究方案, 研究了在硅基二氧化硅表 面不同表面修饰方法对癌肧抗原(CEA)抗体固定的 影响, 以双抗体夹心酶联免疫法(ELISA)评价 CEA 抗体的固定效果, 以期优化篮选出最佳的抗体固定 策略用于蛋白质芯片的进一步开发.

\section{1 实验部分}

\section{1 试剂和仪器}

3-氨基丙基三乙氧基硅烷(APTES, 99\%), 3-巯基 丙基三甲氧基硅烷(MPTS, 85\%, 比利时 ACROS 公 司); 3-缩水甘油醚氧基丙基三甲氧基硅烷(GPTS, 97\%, 美国 Alfa Aesar 公司); 对醛基苯甲酸(FBA, $97 \%$ ), 聚苯乙烯(PS, 平均分子量 25000), 多聚赖氨酸
( $\varepsilon$-PLL, 分子量 70-150 kDa), 蛋白 A(Protein A), 蛋 白 $\mathrm{G}$ (Protein $\mathrm{G}$ ), 亲和素(Avidin), 生物素(D-Biotin, 美国 Sigma-Aldrich 公司); 戊二醛(GA, 25\%( $\varphi$ ) 水溶 液), 2-吗啉乙磺酸(MES, ultra grade), 吐温 20(Tween 20, 美国 Merck 公司); 1-(3-二甲氨基丙基)-3-乙基碳 二亚胺盐酸盐( $\mathrm{EDC} \cdot \mathrm{HCl}, 98 \%), N$-羟基琥珀酰亚胺 (NHS, 98+\%), SMCC(4-(N-马来酰亚胺基甲基)环己 烷-1-羧酸琥珀酰亚胺酯)/sulfo-SMCC (美国 Pierce Biotechnology公司); 癌胚抗原(CEA, 美国Fitzgerald Industries International Inc.公司); 抗癌肧抗原包被抗 体(CEA Ab), 生物素化抗癌胚抗原包被抗体(CEA Ab-Biotin), 辣根酶标记抗癌肧抗原标记抗体(CEA $\mathrm{Ab}-\mathrm{HRP}$ ), TMB 显色液(北京博迈世纪生物技术有 限公司); 牛血清白蛋白(BSA, 98\%), 小牛血清, 羊抗 人 $\operatorname{IgG}$ 纯化抗体 $(\geqslant 95 \%)$, 蔗糖 $(\geqslant 99 \%)$, 海藻糖 $(\geqslant$ 99\%, 北京欣经科生物技术有限公司); 壳聚糖(chitosan, 分子量 $150 \mathrm{kDa}$, 脱乙酰度 $95 \%$ ); 磷酸盐缓冲 液( $\mathrm{PBS}, 3.63 \mathrm{~g} \mathrm{Na}_{2} \mathrm{HPO}_{4} \cdot 12 \mathrm{H}_{2} \mathrm{O}$ 和 $0.24 \mathrm{~g} \mathrm{KH}_{2} \mathrm{PO}_{4}$ 溶 于超纯水并定容至 $1 \mathrm{~L}$ ); 碳酸盐缓冲液 $(\mathrm{CB}, 1.59 \mathrm{~g}$ $\mathrm{Na}_{2} \mathrm{CO}_{3}$ 和 $2.93 \mathrm{~g} \mathrm{NaHCO}_{3}$ 溶于超纯水并定容至 1 L); MES 缓冲液(MES, $15.92 \mathrm{~g} \mathrm{MES}$ 溶于 $900 \mathrm{~mL}$ 超 纯水, 再用 $\mathrm{NaOH}$ 调节 $\mathrm{pH}$ 值到 6.5 , 最后用超纯水 定容至 $1 \mathrm{~L}$ ); 无水乙醇(分析纯), 甲苯(分析纯), $N, N$ 二甲基甲酰胺(DMF, 分析纯, 北京化工厂); 其他试 剂为市售分析纯或更高级别.

EASY pure LF 超纯水系统(美国 Barnstead 公 司); PDC-M 型等离子清洗器(成都铭恒科技发展有 限公司); ZD-85 气浴恒温振荡器(江苏金坛市金城 国胜实验仪器厂); JC2000C 1 全自动接触角仪(上海 中晨数字技术设备有限公司); RT-2100C 酶标分析 仪(深圳市雷杜电子有限公司); 微反应池 $(7.5 \mathrm{~mm} \times$ $13 \mathrm{~mm} \times 5 \mathrm{~mm}$, 自制); 具有 $200 \mathrm{~nm}$ 厚二氧化硅膜 的硅片 $(6.6 \mathrm{~mm} \times 10.7 \mathrm{~mm} \times 0.75 \mathrm{~mm}$, 北京大学微电 子学系).

\section{2 硅片的前处理}

先用无水乙醇和去离子水交替清洗硅片 3 次, 并用氮气流吹干, 然后置于等离子清洗器中处理 2 $\min$ (电压 $600 \mathrm{~V}$, 氧气流量 $800 \mathrm{~mL} \cdot \mathrm{min}^{-1}$ ), 使硅片表 面生成一层活性的羟基(接触角 $<5^{\circ}$ ). 等离子处理后 的硅片应立即进行后续的表面化学修饰, 因为这些 活性的差基是不稳定的, 在空气中容易再次交联形 成硅氧桥键 ${ }^{[32-333}$.

\section{3 硅片表面不同修饰方法固定抗体}




\subsection{1 直接吸附抗体}

将氧等离子体处理好的硅片浸人到 $10 \mathrm{mg} \cdot \mathrm{mL}^{-1}$ PS 的甲苯溶液和 $10 \mathrm{mg} \cdot \mathrm{mL}^{-1}$ PLL 的 PBS 溶液中, 置于室温下浸泡过夜, 然后分别取出. PS 硅片直接 甩干; PLL 硅片用超纯水润洗 5 次, 并用氮气吹干. 然后将两种表面的硅片置于 $50{ }^{\circ} \mathrm{C}$ 烘箱中烘烤 30 min, 取出自然冷却至室温. 将抗癌肧抗原 (CEA)包 被抗体 $\left(10 \mu \mathrm{g} \cdot \mathrm{mL}^{-1}, 0.5 \%\right.$ ( $w$, 质量分数) 海藻糖 ${ }^{[15,34]}$, $\mathrm{CB}$ 溶液)分别滴加到两种硅片表面, 置于 $4{ }^{\circ} \mathrm{C}$ 放置 过夜(如图 1(a)所示).

\subsection{2 直接共价连接抗体}

将氧等离子体处理好的硅片分别迅速浸人到 $5 \%$ ( $\varphi$, 体积分数) APTES 的乙醇溶液, $5 \%(\varphi$, 体积分 数) GPTS 的乙醇溶液, $5 \%(\varphi)$ MPTS 的乙醇溶液中 并置于 $37{ }^{\circ} \mathrm{C}$ 气浴恒温振荡器反应 $2 \mathrm{~h}$. 反应完成后 用无水乙醇清洗 5 次, 并用氮气吹干. 其中 APTES 硅片置于 $90{ }^{\circ} \mathrm{C}$ 烘箱中烘烤 $40 \mathrm{~min}$. 分别得到氨基硅 片, 环氧基硅片和颈基硅片. 然后用四种方式偶联固 定抗体: (1) 直接通过环氧基连接抗体, 将 CEA 包被 抗体 $\left(10 \mu \mathrm{g} \cdot \mathrm{mL}^{-1}, 0.5 \%(w)\right.$ 海藻糖, $\mathrm{PBS}$ 溶液)滴加到 环氧基硅片表面, 然后置于 $4{ }^{\circ} \mathrm{C}$ 放置过夜; (2) 通过 $\mathrm{SMCC}$ 连接抗体, 将颈基硅片浸泡在 $1 \mathrm{mg} \cdot \mathrm{mL}^{-1}$ SMCC 的 DMF 溶液或 sulfo-SMCC 的 PBS 溶液中 $37{ }^{\circ} \mathrm{C}$ 反应 $1 \mathrm{~h}$, 用无水乙醇或 PBS 清洗干净后将 $\mathrm{CEA}$ 包被抗体 $\left(10 \mu \mathrm{g} \cdot \mathrm{mL}^{-1}, 0.5 \%(w)\right.$ 海藻糖, PBS 溶 液)滴加到硅片表面, 然后置于 $4{ }^{\circ} \mathrm{C}$ 放置过夜; (3) 通 过戊二醛连接抗体, 将氨基硅片浸泡在 $5 \%(\varphi)$ 戊二 醛的 PBS 溶液中室温反应 $1.5 \mathrm{~h}, \mathrm{PBS}$ 清洗干净后将 $\mathrm{CEA}$ 包被抗体 $\left(10 \mu \mathrm{g} \cdot \mathrm{mL}^{-1}, 0.5 \%(w)\right.$ 海藻糖, $\mathrm{PBS}$ 溶 液)滴加到硅片表面, 然后置于 $4{ }^{\circ} \mathrm{C}$ 放置过夜; (4) 通 过 EDC/NHS 活化羧基连接抗体, 将氨基硅片浸泡 在 $50 \mathrm{mg} \cdot \mathrm{mL}^{-1}$ 对醛基苯甲酸的 $\mathrm{DMF}$ 溶液中 $37{ }^{\circ} \mathrm{C}$ 反应 $1 \mathrm{~h}$ 得到羧基化表面, DMF 清洗干净后再将羧 基硅片浸泡在 EDC 和 NHS 的混合溶液中 $(0.4 \mathrm{~mol}$ $\mathrm{L}^{-1} \mathrm{EDC}+0.1 \mathrm{~mol} \cdot \mathrm{L}^{-1} \mathrm{NHS}, \mathrm{pH} 6.5, \mathrm{MES}$ 溶液)室温 反应 $30 \mathrm{~min}, 4{ }^{\circ} \mathrm{C}$ 冷藏 $\mathrm{MES}$ 溶液清洗干净后再将 $\mathrm{CEA}$ 包被抗体 $\left(10 \mu \mathrm{g} \cdot \mathrm{mL}^{-1}, 0.5 \%(w)\right.$ 海藻糖, PBS 溶 液)滴加到硅片表面, 然后置于 $4{ }^{\circ} \mathrm{C}$ 放置过夜(如图 1 (b)示).

\subsection{3 加入间隔臂共价连接抗体}

按照 1.3.2 节的方法用 APTES 将硅片表面先 修饰成氨基, 然后将氨基硅片浸泡在 $5 \%(\varphi)$ 戊二醛 的 PBS 溶液中 $2 \mathrm{~h}, \mathrm{PBS}$ 清洗干净后将硅片浸人到 1 $\mathrm{mg} \cdot \mathrm{mL}^{-1} \mathrm{PLL}$ 的 PBS 溶液 $\left(\mathrm{pH}\right.$ 7.4)或 $1 \mathrm{mg} \cdot \mathrm{mL}^{-1}$ 壳 聚糖的醋酸溶液 $(\mathrm{pH}$ 6.5) 中室温反应 $6 \mathrm{~h}, \mathrm{PBS}$ 清洗 干净后再用戊二醛将表面醛基化, 然后将 CEA 包被 抗体 $\left(10 \mu \mathrm{g} \cdot \mathrm{mL}^{-1}, 0.5 \%(w)\right.$ 海藻糖 $\mathrm{PBS}$ 溶液)滴加到 硅片表面, 置于 $4{ }^{\circ} \mathrm{C}$ 放置过夜(如图 1(c)示).

\subsection{4 利用生物亲和作用固定抗体}

按照 1.3 .3 节的方法通过聚赖氨酸和戊二醛将 亲和蛋白 $\mathrm{A} / \mathrm{G}$ 或亲和素 Avidin 固定到硅片表面, PBS 清洗后再将 CEA 包被抗体或 CEA 包被抗体Biotin 滴加到相应的表面, 然后置于 $4{ }^{\circ} \mathrm{C}$ 放置过夜 (如图 1(d)示).

\section{4 抗体芯片的封闭}

将各种 CEA 包被抗体芯片分别浸人到含有 10 $\mathrm{mg} \cdot \mathrm{mL}^{-1} \mathrm{BSA}$ 的 PBS 溶液中, 置于气浴恒温振荡器 上 $37{ }^{\circ} \mathrm{C}$ 温育 $1 \mathrm{~h}$, 然后分别用 $0.1 \%(\varphi)$ PBST 清洗 3 次, 并浸泡在保护剂( $5 \%(w)$ 蔗糖 PBS 溶液)中置于 4 ${ }^{\circ} \mathrm{C}$ 待测. 其中, 通过蛋白 $\mathrm{A} / \mathrm{G}$ 亲和固定的抗体硅片 (蛋白 A/G-CEA 抗体硅片) 需先用羊抗人 $\operatorname{IgG}$ 抗体 进行封闭, 然后再用 BSA 封闭; 通过亲和素-生物素 亲和固定的抗体硅片(Avidin-Biotin-CEA 抗体硅片) 需先用 D-Biotin 进行封闭, 然后再用 BSA 封闭.

\section{5 抗体芯片固定效果的酶联评价}

为了评价硅片表面固定的抗体的密度和生物学 活性, 基于抗体的免疫学特性, 我们采用抗原抗体分 析中常用的酶联免疫夹心反应的方法进行评价(图 2).

这一评价方法可以定量地反映硅片上的抗体固 定效果, 即硅片表面具有生物活性的抗体分布情况. 并且可以移植到其他标志物(如苂光标记或磁标记) 的分析检测中. 具体的操作步骤如下.

\subsection{1 抗原抗体的免疫夹心反应}

将不同固定方法修饰的抗体芯片(各 5 片以上) 清洗干净后, 置于微反应池中, 然后加人不同浓度的 CEA 抗原 $(200 \mu \mathrm{L}, 45 \%(\varphi)$ 小牛血清稀释), 置于气 浴恒温振荡器上 $37{ }^{\circ} \mathrm{C}$ 温育 $1 \mathrm{~h}$, 反应完成后分别取 出用 $0.1 \%(\varphi)$ PBST 清洗 3 遍. 再将硅片放人新的微 反应池中, 接着再加人 CEA 标记抗体-HRP (4 $\mu \mathrm{g}$ $\mathrm{mL}^{-1}, 200 \mu \mathrm{L}, 15 \mathrm{mg} \cdot \mathrm{mL}^{-1} \mathrm{BSA}$ 稀释), 置于 $37{ }^{\circ} \mathrm{C}$ 温 育 $1 \mathrm{~h}$, 最后分别取出用 $0.1 \%(\varphi)$ PBST 清洗 3 遍.

\section{5 .2 显色反应}

将免疫反应后的芯片置于新的微反应池中, 加 人 $\mathrm{TMB}$ 显色液各 $150 \mu \mathrm{L}, 37{ }^{\circ} \mathrm{C}$ 温育 $15 \mathrm{~min}$ 后, 将 显色反应后的溶液移取到 96 孔酶联板中, 再分别加 
(a) Physical adsorption

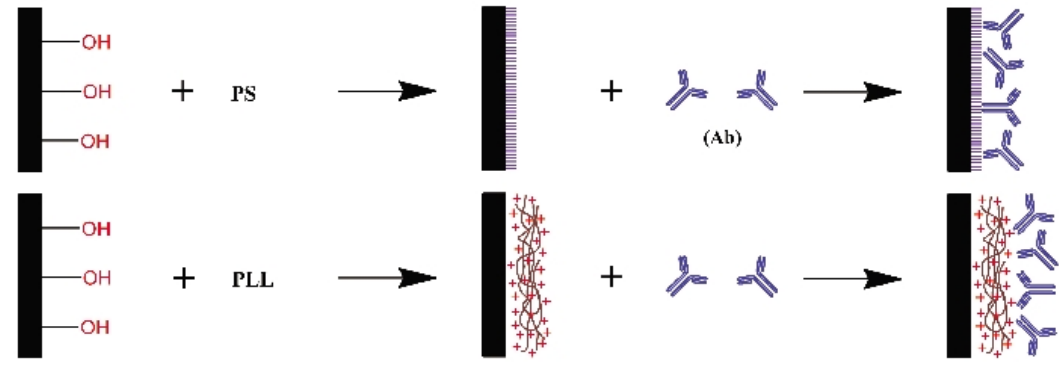

(b) Direct covalent conjugation
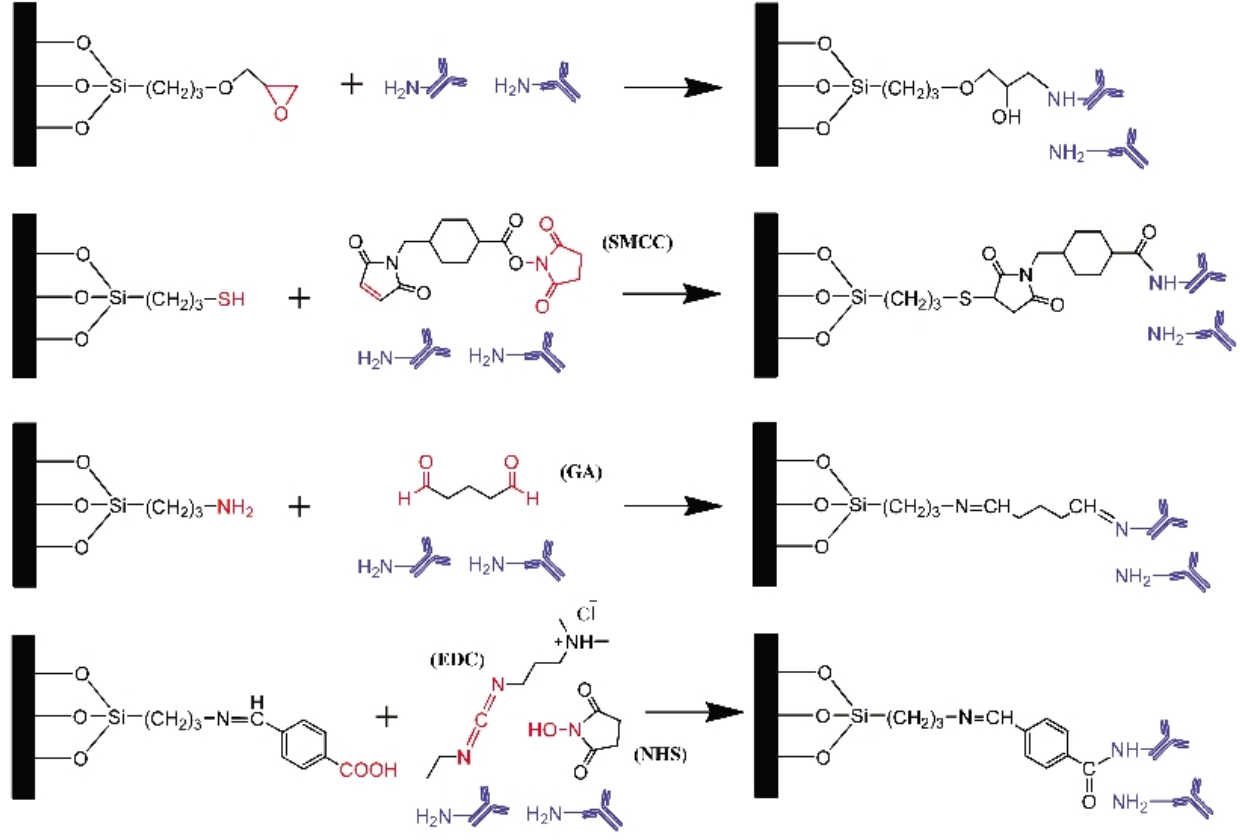

(c) Spacer-added covalent conjugation

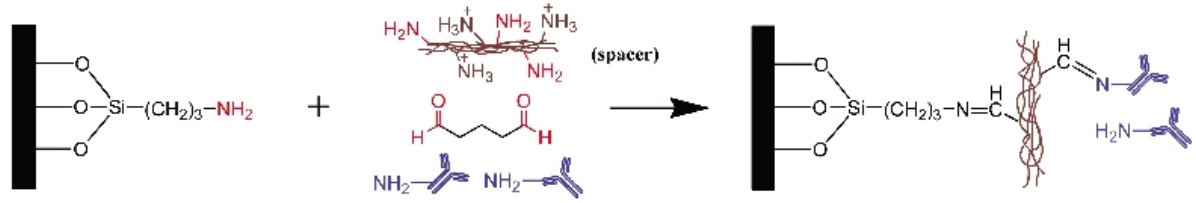

(d) Bioaffinity interaction
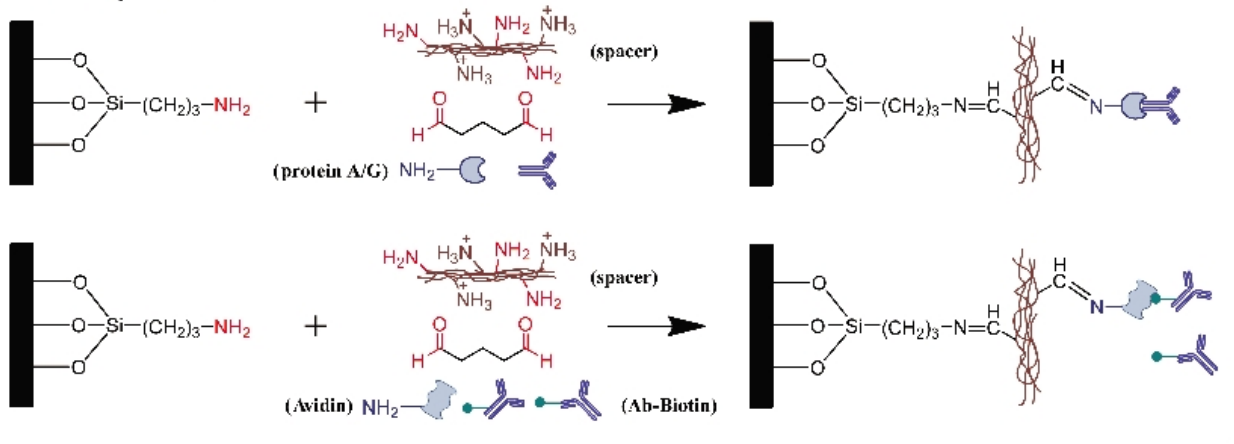

图 1 各种抗体固定方法的硅片表面修饰流程

Fig.1 Procedures of surface modification for various antibody immobilization strategies 

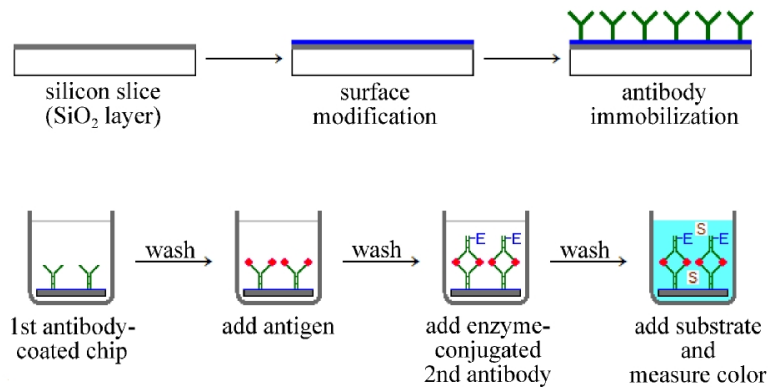

图 2 抗体在硅片上的固定及其酶联免疫夹心评价示意图

Fig.2 Schematic of antibody immobilization and enzyme-linked immunosorbent assay

人终止液 $\left(2 \mathrm{~mol} \cdot \mathrm{L}^{-1} \mathrm{H}_{2} \mathrm{SO}_{4}\right)$ 各 $50 \mu \mathrm{L}$, 最后用酶标仪 进行比色测试, 比色测试应在加人终止液后 $5 \mathrm{~min}$ 内完成.

\section{2 结果与讨论}

\section{1 直接吸附抗体}

蛋白质之间的吸附作用主要来自于疏水作用、 静电作用、范德华力作用、路易斯酸碱力作用以及构 象变化和表面附近受限的侧向扩散 ${ }^{[13]}$. 通过物理吸 附的方式直接固定抗体是最经典的抗体固定方式, 常用的方法是将抗体吸附到疏水性表面(如聚苯乙 烯(PS)表面)或带有高密度电荷的表面(如多聚赖氨 酸(PLL)包被的玻片), 这种方法具有价格低廉、操作 简便的优点. 图 3 是对两种最常用的物理吸附固定 抗体方法的测试结果, 从图中可以看出, 在 PS 表面 随着 CEA 抗原浓度的增加呈现出较好的线性相关, 这说明在 PS 表面通过疏水相互作用可以固定上大 量具有生物活性的 CEA 抗体, 但是却具有较高的非 特异性吸附. 而在 PLL 表面则几乎没有免疫响应,

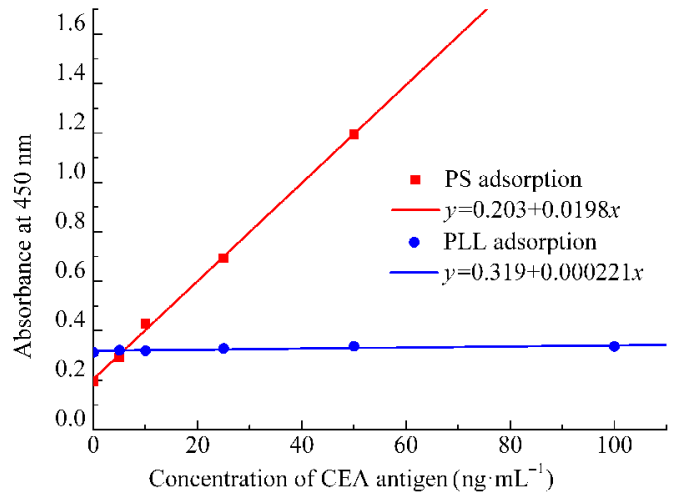

图 3 直接物理吸附固定抗体效果的酶联免疫夹心评价

Fig.3 Evaluate antibody immobilization with physical adsorption by ELISA

PS: polystyrene, PLL: poly-L-lysine
这说明通过静电相互作用并未能有效固定 CEA 抗 体. 我们认为这可能是因为二氧化硅表面主要为一 层电中性的硅差基或硅氧桥键, 很难吸附上电荷富 集的 PLL，因而没有能够形成一层富集电荷的表面 来用于进一步吸附 CEA 抗体. 由于吸附是竞争的和 可逆的, 因此在后续的免疫反应和多次冲洗过程中, 可能造成硅片表面抗体的脱落, 进而影响其结果的 稳定性和一致性, 并且通过物理吸附固定的抗体通 常呈现区域聚集状, 也具有较高的非特异性吸附, 这 在高灵敏度的分析检测中是个很严重的问题, 因而 其应用范围有限.

\section{2 直接共价连接抗体}

相对于物理吸附法固定抗体, 通过氨基、羧基或 颈基共价连接的方式可以使得抗体的固定更加稳 定. 通常的方法是先用硅烷试剂(如 APTES、MPTS 或 GPTS 等)在硅片表面衍生出具有化学活性的功 能团(如氨基、巯基、羧基、环氧基等), 然后再通过各 种偶联试剂(如戊二醛、SMCC、EDC/NHS 等)将抗体 固定到硅片表面. 由于固定效率依赖于交联基团的 数量和偶联反应的效率, 抗体种类的变化和反应条 件的波动常常会带来较大影响, 因而通过直接共价 连接的方式固定抗体的密度常常较低. 图 4 是常用 的几种直接共价连接的方式固定抗体的酶联测试结 果. 从图中可以看出, 利用 APTES 进行硅烷化, 戊 二醛作为偶联试剂具有最好的固定效果, 但是仍然 具有较高的非特异性吸附. 这可能是因为 APTES

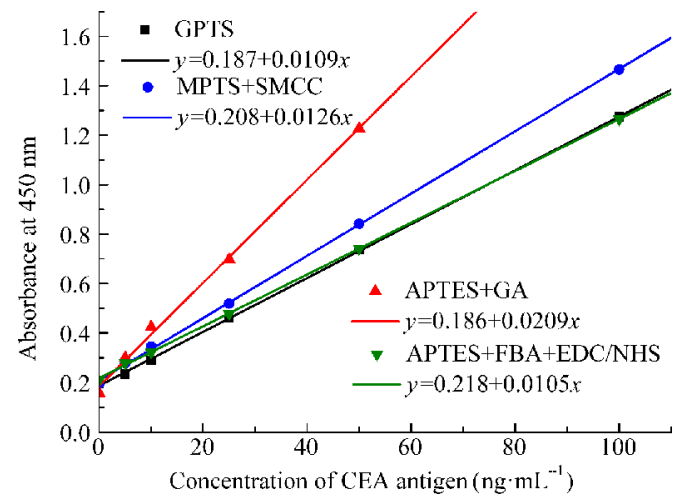

图 4 直接化学共价固定抗体效果的酶联免疫夹心评价

Fig.4 Evaluate antibody immobilization with direct chemical covalent conjugation by ELISA

GPTS: 3-glycidoxypropyl-trimethoxysilane, MPTS: 3-mercaptopropyltrimethoxysilane, SMCC: succinimidyl 4-[N-maleimidomethyl] cyclohexane-1-carboxylate, APTES: 3-aminopropyl-triethoxysilane, GA: glutaraldehyde, FBA: 4-formylbenzoic acid, EDC: 1-(3dimethylaminopropyl)-3-ethylcarbodiimide hydrochloride, NHS: $N$-hydroxysuccinimide 
化的硅片表面形成的是一种致密的毛刷结构, 因而 具有一定的疏水性, 可能带来较高的非特异性吸附. 利用 SMCC 偶联颈基和氨基比通过 EDC/NHS 活化 羧基连接氨基的偶联效果要弱些, 这可能是因为 EDC/NHS 和 SMCC 较为活泼, 在偶联过程中容易 失活或逆平衡解离所致. 而环氧表面对氨基的直接 偶联效果不太好可能是因为环氧基团与氨基的反应 在中性条件下较慢所致 ${ }^{[35]}$, 值得指出的是, 环氧基 团偶联氨基开环之后会生成一个电中性的亲水羟 基, 有助于减弱非特异性吸附.

\section{3 加入不同的间隔臂共价连接抗体}

为了提高共价连接固定抗体的效率, 考虑在固 定抗体之前先在硅片表面引人一层大分子间隔臂, 有文献报道大分子间隔臂的引人可以有效地改善固 相表面的亲水性和生物兼容性, 改善抗原抗体免疫 结合时的空间位阻 ${ }^{[36-37]}$, 提高硅片表面抗体的免疫 反应效率. 我们比较了常用的蛋白质分子 (BSA 和 $\operatorname{IgG}$ )和多氨基聚合物 (PLL 和壳聚糖), 偶联试剂为 戊二醛或 SMCC, 实验结果如图 5 所示.

从图 5 中可以很明显地看出, 多氨基聚合物的 效果远远优于蛋白质分子. 这可能是因为多氨基聚 合物中含有更多的可以参与反应的活性氨基, 因而 具有更高的偶联反应效率. 多聚赖氨酸(PLL)和壳聚 糖(chitosan)均具有很好的固定效果, 其中多聚赖氨 酸表面具有更低的非特异性吸附, 这可能是因为多 聚赖氨酸分子内含有许多亲水功能团, 可以极大地 增强芯片表面的亲水性, 从而起到了降低非特异性 吸附的作用.

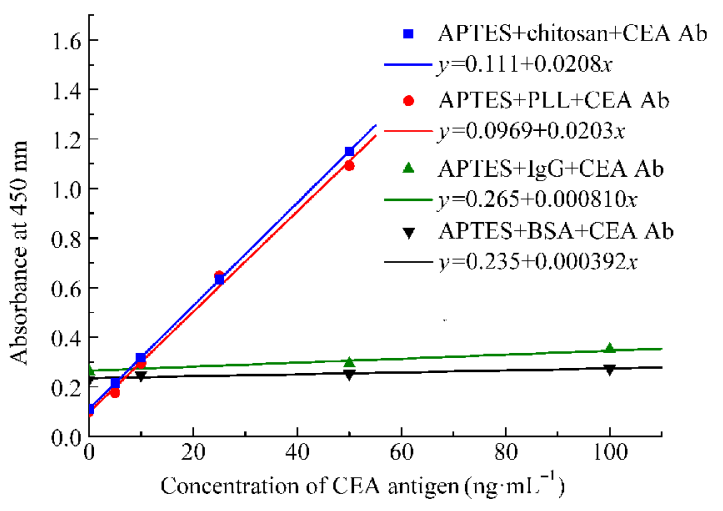

图 5 加入不同间隔臂共价固定抗体效果的酶联免疫夹心 评价

Fig.5 Evaluate antibody immobilization with spaceradded covalent conjugation by ELISA

APTES: 3-aminopropyl-triethoxysilane, PLL: poly-L-lysine, IgG: immunoglobulin, CEA Ab: CEA antibody, BSA: bovine serum albumin

\section{4 利用生物亲和作用固定抗体}

由于参加反应的氨基、羧基或颈基基团与偶联 试剂的共价反应是难以控制的, 如果交联发生在蛋 白质的活性位点上可能会显著降低蛋白质的生物活 性. 为了进一步提高硅片表面固定抗体的生物活性, 并且为了适应各种不同抗体的固定, 我们考虑利用 生物亲和作用的方式固定抗体. 最常用的生物亲和 作用有蛋白 $\mathrm{A} / \mathrm{G}-\mathrm{IgG}$ 抗体( $\mathrm{Fc}$ 段)亲和作用和AvidinBiotin 亲和作用 ${ }^{[17,31,38]}$. 在本实验中, 我们分别将蛋白 A、蛋白 G、Avidin 分子共价固定到硅片表面, 再利用 生物亲和作用将 CEA 抗体(IgG 抗体)或 CEA 抗体Biotin 固定到硅片表面, 然后分别进行酶联免疫测 试评价抗体固定效果, 实验结果如图 6 所示.

从图 6 中可以看出, 通过生物亲和作用可以有 效地固定抗体, 并且具有较高的生物活性, 这可能是 因为蛋白 $\mathrm{A}$ 、蛋白 $\mathrm{G}$ 或 Avidin 分子承受了固相表面 的微环境变化和共价交联的化学变化而非抗体本 身, 并且借助生物亲和作用可以实现抗体的定向固 定, 因而最大程度地保留了抗体特异结合位点的生 物活性和空间取向 ${ }^{[10,17,39-41]}$. 同时我们发现, 这种利用 生物亲和作用固定抗体的方法具有较高的非特异性 吸附. 这可能是因为这些生物亲和表面不能有效地 封闭造成的. 特别是 Avidin 表面, 由于固定的是生 物素化的包被抗体, 而生物素是电子富集的小分子, 在抗体上的标记通常是随机并且大量的 (一个抗体 分子上通常连接有几个到十几个 Biotin 小分子), 因 而对后续的 CEA 标记抗体-HRP 分子具有较强的静 电吸附作用, 解决这个问题的方法之一是对包被抗

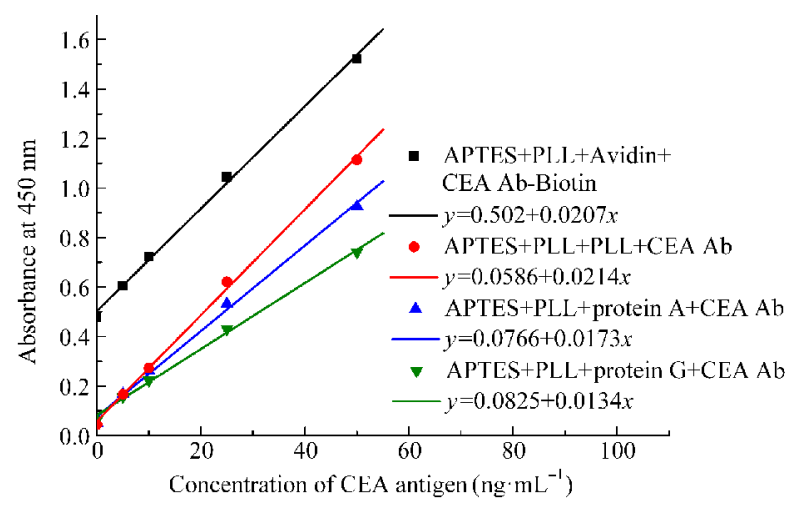

图 6 利用生物亲和作用固定抗体效果的酶联免疫 夹心评价

Fig.6 Evaluate antibody immobilization with biological affinities by ELISA

APTES: 3-aminopropyl-triethoxysilane, PLL: poly-L-lysine, CEA Ab-Biotin: biotinylated CEA antibody 
体进行位点特异性生物素化 ${ }^{[42]}$. 而共价连接两层聚 赖氨酸(PLL)的表面可以进一步提高固定效率和降 低非特异性吸附.

\section{3 结 论}

实验结果表明, 通过共价连接固定抗体可以得 到比通过物理吸附更好的固定效果, 各种偶联反应 中成二醛对氨基的偶联效率最高. 引人含有多功能 团的聚合物分子可以提高偶联固定的反应效率, 多 聚赖氨酸和壳聚糖是很好的氨基放大试剂, 可以用 于氨基偶联试剂(如戊二醛)的增强固定, 减弱非特 异性吸附, 并且可以增加抗体分子与平面硅片之间 的距离, 增加固定抗体的柔韧度, 改善空间取向, 从 而提高硅片表面固定抗体的生物活性. 对于 $\mathrm{IgG}$ 类 型的某些抗体, 可以利用蛋白 $\mathrm{A}$ 或蛋白 $\mathrm{G}$ 的亲和作 用进行定向固定, 最大限度地保持固定抗体的生物 活性.

\section{References}

1 Zhu, H.; Snyder, M. Curr. Opin. Chem. Biol., 2001, 5: 40

2 MacBeath, G. Nat Genet, 2002, 32(suppl.): 526

3 Templin, M. F.; Stoll, D.; Schrenk, M.; Traub, P. C.; Vöhringer, C. F.; Joos, T. O. Trends Biotechnol., 2002, 20: 160

$4 \mathrm{Ng}$, J. H.; Ilag, L. L. Journal of Cellular and Molecular Medicine, 2002, 6: 329

5 Wilson, D. S.; Nock, S. Angew. Chem. Int. Edit., 2003, 42: 494

6 Angenendt, P. Drug Discovery Today, 2005, 10: 503

7 Kusnezow, W.; Hoheisel, J. D. Biotechniques, 2002, 33(suppl.): 14

8 Liotta, L. A.; Espina, V.; Mehta, A. I.; Calvert, V.; Rosenblatt, K.; Geho, D.; Munson, P. J.; Young, L.; Wulfkuhle, J.; Petricoin III, E. F. Cancer Cell, 2003, 3: 317

9 Lutanie, E.; Voegel, J. C.; Schaaf, P.; Freund, M.; Cazenave, J. P.; Schmitt, A. Proc. Natl. Acad. Sci. U. S. A., 1992, 89: 9890

10 Vijayendran, R. A.; Leckband, D. E. Anal. Chem., 2000, 73: 471

11 Rossier, J. S.; Gokulrangan, G.; Girault, H. H.; Svojanovsky, S.; Wilson, G. S. Langmuir, 2000, 16: 8489

12 Angenendt, P.; Glökler, J.; Murphy, D.; Lehrach, H.; Cahill, D. J. Anal. Biochem., 2002, 309: 253

13 Kusnezow, W.; Hoheisel, J. D. J. Mol. Recognit., 2003, 16: 165

14 Seong, S. Y. Clin Diagn Lab Immunol, 2002, 9: 927

15 Kusnezow, W.; Jacob, A.; Walijew, A.; Diehl, F.; Hoheisel, J. D. Proteomics, 2003, 3: 254

16 Niemeyer, C. M.; Wacker, R.; Adler, M. Nucl. Acids Res., 2003, 31: e90

17 Peluso, P.; Wilson, D. S.; Do, D.; Tran, H.; Venkatasubbaiah, M.; Quincy, D.; Heidecker, B.; Poindexter, K.; Tolani, N.; Phelan, M.; Witte, K.; Jung, L. S.; Wagner, P.; Nock, S. Anal. Biochem., 2003, 312: 113
18 Ramachandran, N.; Hainsworth, E.; Bhullar, B.; Eisenstein, S.; Rosen, B.; Lau, A. Y.; Walter, J. C.; LaBaer, J. Science, 2004, 305: 86

19 Oh, S. J.; Hong, B. J.; Choi, K. Y.; Park, J. W. OMICS, 2006, 10: 327

20 Iwata, R.; Satoh, R.; Iwasaki, Y.; Akiyoshi, K. Colloids Surf. B, 2008, 62: 288

21 Hermanson, G. T. Bioconjugate techniques. 2nd ed. Rockford, Illinois: Academic Press, 2008

22 Haab, B. B.; Dunham, M. J.; Brown, P. O. Genome Biol., 2001, 2 0004.1

23 Stillman, B. A.; Tonkinson, J. L. Biotechniques, 2000, 29: 630

24 Qian, W.; Yao, D.; Yu, F.; Xu, B.; Zhou, R.; Bao, X.; Lu, Z. Clin. Chem., 2000, 46: 1456

25 MacBeath, G.; Schreiber, S. L. Science, 2000, 289: 1760

26 Piehler, J.; Brecht, A.; Geckeler, K. E.; Gauglitz, G. Biosens. Bioelectron., 1996, 11: 579

27 Penzol, G.; Armisén, P.; Fernández-Lafuente, R.; Rodés, L.; Guisán, J. M. Biotechnol. Bioeng., 1998, 60: 518

28 Afanassiev, V.; Hanemann, V.; Wolfl, S. Nucl. Acids Res., 2000, 28: e66

29 Piletsky, S. A.; Matuschewski, H.; Schedler, U.; Wilpert, A.; Piletska, E. V.; Thiele, T. A.; Ulbricht, M. Macromolecules, 2000, 33: 3092

30 Wilson, D. S.; Nock, S. Curr. Opin. Chem. Biol., 2002, 6: 81

31 Neubert, H.; Jacoby, E. S.; Bansal, S. S.; Iles, R. K.; Cowan, D. A.; Kicman, A. T. Anal. Chem., 2002, 74: 3677

32 Gupta, B.; Plummer, C.; Bisson, I.; Frey, P.; Hilborn, J. Biomaterials, 2002, 23: 863

33 Stine, R.; Cole, C. L.; Ainslie, K. M.; Mulvaney, S. P.; Whitman, L. J. Langmuir, 2007, 23: 4400

34 Gibson, T. D.; Woodward, J. R. Protein stabilization in biosensor systems//Biosensors and chemical sensors. Washington, DC: American Chemical Society, 1992: 40

35 Mateo, C.; Torres, R.; Fernández-Lorente, G.; Ortiz, C.; Fuentes, M.; Hidalgo, A.; López-Gallego, F.; Abian, O.; Palomo, J. M.; Betancor, L.; Pessela, B. C. C.; Guisan, J. M.; Fernández-Lafuente, R. Biomacromolecules, 2003, 4: 772

36 Lee, S. D.; Hsiue, G. H.; Chang, P. C. T.; Kao, C. Y. Biomaterials, 1996, 17: 1599

37 Kato, K.; Ikada, Y. Biotechnol. Bioeng., 1995, 47: 557

38 Lee, W.; Oh, B. K.; Bae, Y. M.; Paek, S. H.; Lee, W. H.; Choi, J. W. Biosensors and Bioelectronics, 2003, 19: 185

39 Turková, J. J. Chromatogr. B, 1999, 722: 11

40 Jung, Y.; Lee, J. M.; Jung, H.; Chung, B. H. Anal. Chem., 2007, 79: 6534

41 Völimaa, L. Streptavidin—a versatile binding protein for solidphase immunoassays [D]. Turku: University of Turku, 2008

42 Lue, R. Y. P.; Chen, G. Y. J.; Zhu, Q.; Lesaicherre, M. -L.; Yao, S. Q. Site-specific immobilization of biotinylated proteins for protein microarray analysis//Protein arrays. Totowa: Humana Press Inc., 2004: 85 